\title{
Modelling the Ability of Rheoencephalography to Measure Cerebral Blood Flow
}

\author{
Konstantin S. Brazovskii ${ }^{1}$, Jacov S. Pekker ${ }^{2}$ and Oleg S.Umanskii ${ }^{2}$ \\ 1. Deparment of Industrial and Medical Electronics, Tomsk Polytechnic University, Tomsk, Russia \\ 2. Department of Medical and Biological Cybernetics, Siberian State Medical University, Tomsk, Russia \\ 3. E-mail any correspondence to: mbc@tpu.ru
}

\begin{abstract}
Despite the long history of rheoencephalography (REG), some important aspects of the method are still debatable. Bioimpedance measurements offer great potential benefit for study of the human brain, but the traditional four or six electrode method suffers from potential misinterpretations and lack of accuracy. The objective of this paper is to study the possible mechanism of REG formation by means of numerical modelling using a realistic finite element model of the human head. It is shown that the cardiac related variations in electrical resistivity of the scalp contributes more than $60 \%$ to the REG amplitude, whereas the brain and cerebrospinal fluid are mutually compensated by each over.
\end{abstract}

Keywords: Rheoenchephalography, finite element model, numerical modelling

\section{Introduction}

Electrical rheoencephalography is a well known electrophysiological method, which is intended for estimating the cerebral blood flow (CBF) by means of noninvasive electrical impedance measurements made through scalp electrodes. Presumably, the origin of REG is a pulsed variation of the brain conductivity during the cardiac cycle. Due to the fact that REG is widely used in routine clinical studies and has a long history of practical applications, the ability of REG to assess the functional state of the human brain has been well investigated. Despite this fact, the method is frequently referred to as unreliable and controversial because the measurements are ambiguous with a high risk of clinical misinterpretation [1].

The most controversial characteristic of REG is its ability to evaluate correctly the CBF from the surface electrodes through the anatomical structures of the human head. The argument is that a weak alternating electrical current, which is used for the impedance measurements, is shunted by the scalp with its low electrical conductivity, and can not reach the brain [2]. Another delicate feature of REG is its dependence on individual variations in the anatomy of the human head. Furthermore, small changes in the positions of the electrodes lead to a significant deviation in the measured impedance. One more disadvantage is a relatively complicated procedure that includes the positioning of the electrodes on the head, special patient treatment in advance, and, as a consequence of high sensitivity to dislocations, reducing movements of the head during the test. A straightforward interpretation of the measured values is also debatable.
These are the reasons why REG is not the method of choice in clinical studies of CBF. Moreover, having no visualization ability, REG can not compete with modern computer tomography systems, functional magnetic resonance imaging machines, Doppler effect based ultrasound equipment and radioactive isotopic imaging. Nevertheless, REG still remains attractive on account of its simplicity [3]. In addition, new bioimpedance measurement techniques, such as electrical impedance tomography of the brain, provide enough sensitivity and accuracy to register small changes in electrical conductivity of the head tissues during the cardiac cycle, but the origin of these signals should be studied thoroughly to avoid uncertainty of interpretation.

Most comprehensive clinical, laboratory and theoretical studies of REG were conducted between 1960-1980 [4]. Since that period, several models of REG formation have been developed, but, to a certain extent, contradict the laboratory and clinical observations. First of all, the common approach [3] uses a traditional physiological experimental technique with laboratory animals under the very specific conditions of an acute surgical operation. Obviously, these conditions differ significantly from the normal state and results should be interpreted carefully. Taking into account the ethical issues, such experiments have serious limitations and create a strong objection within society. The second argument, frequently used against the acute experiments, concerns the blood circulation under these particular circumstances. It is absolutely necessary to provide an experimental animal with appropriate anaesthesia and blood circulation during the whole operation. It is absolutely necessary to provide an experimental animal with appropriate anaesthesia. Unfortunately, anesthetic agents noticeably interfere with cerebral blood flow autoregulation [5] and bioimpedance measurements, changing the concentration of ions in the blood and physiological properties of vessels.

In order to avoid possible inaccuracy, we used an approach based on a realistic mathematical model of the human head. This paper presents the model which was developed to fit the specific requirements of bioimpedance measurements, the results of numerical experiments with the model, and possible mechanism of REG formation.

\section{Materials and methods}

A realistic model of the human head was built using the fuzzy models from the simulated brain dataset (the SBD, [6]). The dataset was preprocessed in order to remove some artefacts, and the image was blurred with a 3D Gaussian filter. After 
that a triangulated surface of the head was reconstructed by means of a surface deformation technique. Several specific conditions were applied to the surface reconstruction method to guarantee smoothness, good quality of triangles, and absence of self-intersecting regions. The surface model consists of 10242 nodes and 20480 surface elements (triangles). A volume tetrahedral mesh was obtained with TetGen software [7] using the surface mesh as a boundary description. The final model was made of 57215 nodes and 261827 finite tetrahedra elements. Next, all finite elements were assigned to the electrical conductivity and permittivity values according to the amount of the particular tissue inside the tetrahedra (1).

$$
\begin{array}{r}
\sigma_{m}(f)=\sum_{i=1}^{12} w_{i} \cdot \sigma_{i}(f), \\
\varepsilon_{m}(f)=\sum_{i=1}^{12} w_{i} \cdot \varepsilon_{i}(f), \\
w_{i}=\frac{N_{i}}{\sum N_{i}}, i=1 . .12
\end{array}
$$

Here $\sigma_{m}(f)$ and $\varepsilon_{m}(f)$ are conductivity and permittivity of the $m$ th finite element at frequency $f, w_{i}$ is a contribution of the particular tissue into the electrical values, $\sum w_{i}=1, N_{i}$ is the number of pixels with the same type of tissue inside the finite element, $i$ identifies the kind of the tissue. The original SBD model involves twelve different types of tissue (cerebral spinal fluid (CSF), grey and white matter, skin, muscles, etc.). We used the electrical parameters $\sigma_{i}(f)$ and $\varepsilon_{i}(f)$ from Database of Tissue Properties [8]. The frequencies were chosen within a range of $50-200 \mathrm{kHz}$ as the most common for REG. Finally, the virtual surface electrodes [6] with the approximate area $300 \mathrm{~mm}^{2}$ and the contact resistance 250 Ohms at $100 \mathrm{kHz}$ were placed in the "10/20" system standard locations. In numerical experiments we used a subset of the whole system (F3, F4, T3, T4, T7, T8, O1, O2), according to the common REG scheme. The amplitude of excitation current was $1 \mathrm{~mA}$. The voltages were calculated between pairs F3-T3, T3-T7, T7-O1 and F4-T4, T4-T8, T8-O2. Four electrode measurement protocol with the complete electrode model was applied.

The numerical experiments included calculation of the background impedance with the initial values of $\sigma(f)$ and $\varepsilon(f)$, then the parameters were slightly changed and the impedance was computed again.

We proposed a method to model the small variations in electrical parameters caused by a pulsed circulation of fluids inside the scull. The idea behind this method is based on the simple fact that the resistance depends on resistivity and size of the object (2).

$$
R=\int_{0}^{l} \frac{\rho(l)}{A(l)} d l
$$

$R$ is the resistance, $\rho(l)$ is the resistivity, $A(l)$ is a cross section of the object, $l$ is a line of integration. Thus, in the case of very small mechanical deformations of the brain we can model the $\Delta R$ changing only $\rho(l)$ instead of exact calculation $A(l)$ and $l$. A similar equation also exists for the capacitance. The changes in the electrical parameters were calculated using (1) with modified weights $w_{i}$ for the

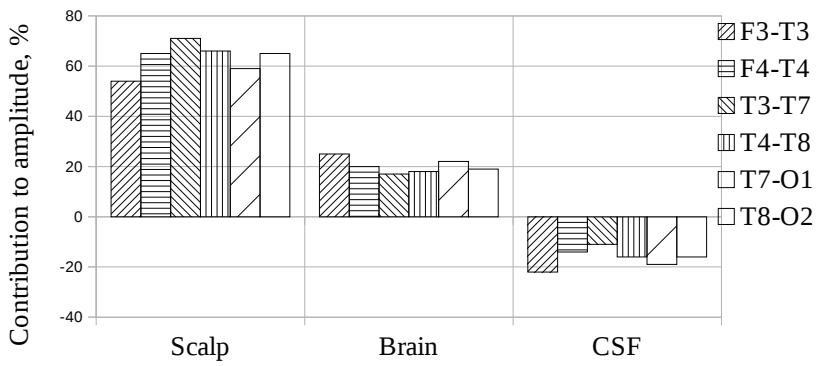

Fig. 1: Contribution of the brain, skin, and CSF into the REG's amplitude

brain, cerebral spinal fluid, and blood. These modifications were applied only to the finite elements lying on the borders between the brain and fluids.

To calculate the impedance $Z$ a forward problem (3) with the complete electrodes model [9] was solved.

$$
\begin{array}{r}
\nabla \cdot(\sigma+j \varepsilon) \nabla \varphi=0 \text { in } \Omega, \\
\vec{n} \cdot(\sigma+j \varepsilon) \nabla \varphi=0 \text { on } \partial \Omega \backslash \Gamma_{e}, \\
\varphi+z \vec{n} \cdot(\sigma+j \varepsilon) \nabla \varphi=U \text { on } \Gamma_{e}, \\
I_{e}=\frac{1}{S_{e}} \int_{S_{e}} \vec{n} \cdot(\sigma+j \varepsilon) \nabla \varphi d S, 1 \leq e \leq N e
\end{array}
$$

$\varphi$ is electrical potential, $\vec{n}$ is a normal vector to the surface $\partial \Omega, \Gamma_{e}$ is a surface of the electrode $e, z$ is its contact impedance, $U$ is a voltage (complex value) at the electrode $e$. As a result of solving (3), the complex voltages $U_{e_{l} e_{k}}$ and the electrical impedance $Z$ between pairs $e_{l} e_{k}$ of electrodes were calculated as $Z_{e_{l} e_{k}}=U_{e_{l} e_{k}} / I$.

\section{Results}

The contributions made by the tissues to the voltages at the surface electrodes were calculated considering a linear approximation of a sensitivity matrix. The sensitivity matrix, or Jacobian $J$, gives the forward solution (3) as:

$$
\begin{array}{r}
J_{e m}=\frac{\partial U_{e}}{\partial \sigma_{m}^{*}} ; \\
U_{e}=\sum_{m=1}^{M} J_{e m} \cdot \sigma_{m}^{*}= \\
\sum_{m=1}^{M} J_{e m} \cdot \sum_{i} w_{i m} \cdot \sigma_{i m}^{*}
\end{array}
$$

where $\sigma^{*}=(\sigma+j \varepsilon), M$ a number of finite elements in the model, $U_{e}$ the voltage at the electrode $e$. Consequently, a partial sum in (4) may be used to find the contribution of a particular tissue to the aggregated signal. The calculated values for some electrode's pairs are given in Fig.1. The percentages describe parts of $U_{e}$ caused by the different tissues.

The next numerical experiment was conducted in order to compute the influence of small changing in the electrical parameters on the voltages at the surface electrodes. As described above, the weights $w_{i}$ of the skin, brain, blood and 


\begin{tabular}{c|cccccc} 
& F4-T4 & F3-T3 & T4-T8 & T3-T7 & T8-O2 & T7-O1 \\
\hline Rbg, Ohm & 529.264 & 477.82 & 552.155 & 507.325 & 560.549 & 533.523 \\
\hline R scalp, Ohm & 529.116 & 477.703 & 551.963 & 507.142 & 560.349 & 533.282 \\
$\Delta$ R, Ohm & -0.148 & -0.117 & -0.192 & -0.183 & -0.2 & -0.241 \\
\hline R brain, Ohm & 529.218 & 477.766 & 552.108 & 507.275 & 560.475 & 533.454 \\
$\Delta$ R, Ohm & -0.046 & -0.054 & -0.047 & -0.05 & -0.074 & -0.069 \\
\hline R CSF, Ohm & 529.296 & 477.867 & 552.185 & 507.368 & 560.612 & 533.584 \\
$\Delta$ R, Ohm & 0.032 & 0.047 & 0.03 & 0.043 & 0.063 & 0.061 \\
\hline Amplitude, Ohm & -0.162 & -0.124 & -0.209 & -0.19 & -0.211 & -0.249 \\
\hline
\end{tabular}

Tab. 1: Calculated amplitudes of REG

CSF were varied, saving $\sum w_{i}=1$. The variations correspond to the pulsatile intracranial volume (ICV) [10]. The value given there is about $1 \mathrm{ml}$, or $0.1 \%$ of the whole ICV. It is also important to note, that there is a small delay, approximately $200 \mathrm{~ms}$ [10], between the peak inflow of arterial blood and outflow of CSF. Hence, at least three time points of the cardiac cycle should be modelled: background, the arterial blood volume inflow peak, and the CSF volume outflow peak. The results are given in Tab.1. All values are a module of the calculated impedance. $\mathrm{R}_{b g}$ corresponds to the background distribution of the conductivity and permittivity. $\mathrm{R}_{\text {scalp }}$ and $\Delta \mathrm{R}_{\text {scalp }}, \mathrm{R}_{\text {brain }}$ and $\Delta \mathrm{R}_{\text {brain }}, \mathrm{R}_{C S F}$ and $\Delta \mathrm{R}_{C S F}$ are mutual resistances and their variations due to pulsatile changes in the electrical parameters of the corresponding tissue.The electrical impedance of the skin changes simultaneously with the impedance of the brain because the arterial blood inflow, thus the amplitudes $\Delta \mathrm{R}_{\text {scalp }}$ and $\Delta \mathrm{R}_{\text {brain }}$ may be summarised. The negative values mean that the calculated resistance becomes lower on the peak of the arterial blood inflow. The CSF outflow increases the resistance and contributes a small positive value, comparable to the absolute $\Delta \mathrm{R}$ of the brain.

\section{Discussion}

As a result of the numerical experiments a reasonable hypotheses concerning the mechanism of REG formation has been proposed. Actually, our numerical results to a certain extent prove the widespread point of view that the main source of REG is a pulsatile change in electrical impedance of the scalp. Although the volume of the scalp is significantly lower in comparison with the brain, the scalp generates a signal that is 3 or 4 times stronger than the signal of the brain because so called "near electrode effects". Current density near the electrodes is higher; consequently, it creates a higher voltage across the adjacent regions. This effect can partially be reduced by the four electrode method, but it deals mainly with the contact resistance of the electrode. We think that is a reason why the standard low electrodes count REG can not distinguish between intra- and extracranial sources of pulsation.

Another point of discussion concerns the method of modelling small variations in the electrical impedance. We used this approach to calculate new values of the electrical parameters because, according to the Monro-Kellie relation, the internal volume of the skull remains constant during the cardiac cycle, so the relative volumes of the brain, blood, and CSF should vary. These variations can be modelled either using elasticity theory [11], or by direct modification of the electrical parameters of the finite elements. The complexity of the mechanical model of the brain, which takes into account elasticity and fluid flow, is very high. Moreover, the mechanical models are also based on tomographic images with a spacial resolution of about $1 \mathrm{~mm}$, whereas the deformation of the anatomical structures during the cardiac cycle is very small and can not be detected even on the high resolution images. Besides computational complexity, the realistic mechanical model introduces one more source of errors as the mechanical parameters of the brain (mainly elasticity) are extremely difficult to be measured precisely. In case of very small (no more than $0.1 \%$ of initial volume) elastic deformation of the brain the borders between anatomical structures move only slightly from the original positions and likely remain inside the same finite elements. Therefore, both approaches seem to be equal, taking into account the estimated accuracy of the final solution.

It is also clear that changes in the electrical impedance of the brain are closely linked to its compliance; intracranial pressure plays an important role as well.

\section{Conclusion}

Rheoencephalography as a simple and completely noninvasive method is very attractive. Unfortunately, the inherited features of REG, related to the physical limitations of bioimpedance measurements, are crucial in context of quantitative measurements of the CBF. The results of our numerical experiments may explain some controversial aspects of REG along with the reasons for misinterpretation. From the point of view of this particular work, the variety of existing clinical and experimental findings could be explained by the fact that the amplitude of REG is a non-linear function of the heart's stroke volume, the brain's compliance, intracranical pressure, and cerebral blood flow. Under certain circumstances (high compliance, high stroke volume, and low intracranial pressure), CBF may create a strong signal, but gen- 
erally these factors are unknown because they can be measured only by means of MRI. Obviously, it it not necessary any more to record REG having a completed MRI study.

\section{Acknowledgements}

This work is funded within the framework of realization of Strategic Programme on National Research Tomsk Polytechnic University Competitiveness Enhancement in the Group of Top Level World Research and Academic Institutions.

\section{References}

1. Bodo M. Studies in Rheoencephalography (REG). Review article. Journal Electrical Bioimpedance. 2010;1:18-40. http://dx.doi.org/10.5617/jeb.109.

2. Perez JJ, Guijarro E, Ortiz P, Pons JM. New Perspectives in Rheoencephalography. Medical Information Science Reference; 2008. p. 990-995. http://dx.doi.org/10.4018/978-1-59904-889-5.ch123.

3. Moskalenko YU, Weinstein G, Masalov I, Halvorson P, et al. Multifrequency REG: Fundamental Background,Informational Meaning and Ways of Data Analysis and Automation. American Journal of Biomedical Engineering. 2012;2(4):163-174. http://dx.doi.org/10.5923/j.ajbe.20120204.03.

4. Moskalenko YU. Biophysical aspects of cerebral circulation. Pergamon Press; 1980.

5. Kazuto M, Iwao K. Anesthesia and the quantitative evaluation of neurovascular coupling. Journal of Cerebral Blood Flow and Metabolism. 2012;32(7):1233-1247. http://dx.doi.org/10.1038/jcbfm.2012.50.

6. McGill Centre for Integrative Neuroscience. BrainWeb: Online Interface to a 3D MRI Simulated Brain Database;. Available from http://www.bic.mni.mcgill.ca/brainweb/. Cited 2014 Oct 27.

7. Hang S. TetGen: A Quality Tetrahedral Mesh Generator and Three-Dimensional Delaunay Triangulator;. Available from http://wias-berlin.de/software/tetgen/. Cited 2013 Nov 4.

8. Hasgall PA, Neufeld E, Gosselin MC, Klingenböck A, Kuster $\mathrm{N}$. It is Database for thermal and electromagnetic parameters of biological tissues Version 2.5; . Available from http://www.itis.ethz.ch/database. Cited August 1st, 2014.

9. Nyvonen N. Complete electrode model of electrical impedance tomography: approximation properties and characterization of inclusions. SIAM Journal Applied Mathematics. 2004;64(3):902-931. http://dx.doi.org/10.1137/S0036139903423303.

10. Alperin N, Mazda M, Lichtor T, Lee SH. From Cerebrospinal Fluid Pulsation to Noninvasive Intracranial Compliance and Pressure Measured by MRI Flow Studies. Current Medical Imaging Reviews. 2006;(2):117-129. http://dx.doi.org/10.2174/157340506775541622.

11. Kim DJ, Czosnyka Z, Kasprowicz M, Smieleweski P, Baledent $\mathrm{O}$, Guerguerian AM, et al. Continuous monitoring of the Monro-Kellie doctrine: is it possible? Journal of neurotrauma. 2012;29(7):1354-1363. http://dx.doi.org/10.1089/neu.2011.2018. 